\title{
Monoklonale Antikörper bei allergischem Asthma
}

\author{
Bei schwerem allergischen Asthma bronchiale halbiert sich \\ durch die Komedikation mit einer monoklonalen Immun- \\ therapie der Kortisonbedarf. Dies hat die randomisierte und \\ doppeltblinde Studie des internationalen Forscherteams um \\ E. H. Bel nun demonstriert. \\ N Engl J Med 2014; 371: 1189-1197
}

\section{Fazit}

Beim allergischen Asthma bronchiale mit deutlicher Eosinophilie sollte nach Angaben der Autoren der adjuvante Einsatz des monoklonalen Antikörpers Mepolizumab erwogen werden. So kann die notwendige systemische Kortisondosis deutlich reduziert werden. Die ersten klinischen Befunde zeigen, dass durch eine solche Doppeltherapie nicht nur Kortison eingespart wird, auch die Asthmasymptomatik verbessert sich. Dies gelingt, ohne den Patienten durch weitere Nebenwirkungen zu belasten.

Dr. Horst Gross, Berlin
Beim schweren, allergisch bedingten Asthma bronchiale ist Kortison die therapeutische Ultima Ratio. Aus Furcht vor Nebenwirkungen wird dabei oft suboptimal dosiert. Um dieses Dilemma zu umgehen, bietet sich der adjuvante Einsatz von monoklonalen Antikörpern an. Diese wirken über die Blockierung von Interleukin-5 zusätzlich antiinflammatorisch. Das könnte die Effektivität des eingesetzten Kortisons entscheidend verbessern und so die $\mathrm{Ne}$ benwirkungen minimieren. Ob sich dieser Ansatz unter klinischen Bedingungen bewährt, bedurfte noch einer empirischen Überprüfung.

Bei den 135 Patienten der randomisierten, doppelt-verblindeten Studie bestand für mindestens 6 Monate die Notwendigkeit einer systemischen Kortisontherapie. Die begleitende Eosinophilie lag über einem Schwellenwert von 300 Zellen pro $\mu$ l Blut. Vor Studienbeginn war die Kortisontherapie anhand klinischer Kriterien optimiert worden. Randomisiert erhielten die Patienten dann zusätzlich entweder für 20
Wochen den Antikörper Mepolizumab subkutan oder eine Placebo-Injektion. Primärer Outcomeparameter war das Ausmaß der Kortisonreduktion, die durch Mepolizumab erzielt wurde. Als Vergleichspunkt diente die Kortisondosis nach der 20. Studienwoche in Relation zur optimierten Ausgangsdosis. Als sekundäre Parameter erfassten die Autoren die Rate der Exazerbationen, das subjektive Befinden der Patienten und die Nebenwirkungsprofile von Kortison und Mepolizumab.

Der Einsatz des Antikörpers reduziert mit hoher Wahrscheinlichkeit (Odds Ratio 2,39) die notwendige Kortisondosis. Im Median gelang es in der Antikörpergruppe die Kortisondosis um 50\% zu senken. Die Rate der Exazerbationen verminderte sich in der Antikörpergruppe um 32\%. Unter Mepolizumab zeigte sich auch im Asthma Control Questionnaire eine deutliche Verbesserung der Asthmabeschwerden. Die unter der Antikörpertherapie erfassten Nebenwirkungen entsprachen denen unter Placebo.

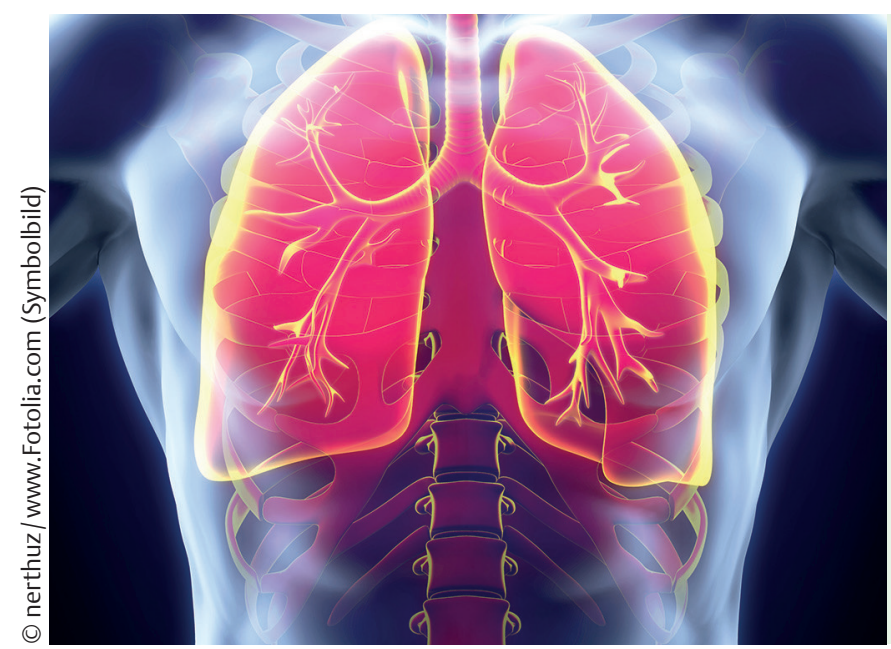

Für Patienten mit schwerem Asthma und nachweisbaren eosinophilen Granulozyten im Blut besteht mit Mepolizumab eine neue Therapieoption. Die Behandlung mit dem monoklonalen Antikörper scheint dabei sicher zu sein, denn es waren keine wesentlichen Nebenwirkungen nachweisbar.

\section{Ausschreibung \\ Förderpreis im Bereich Allergologie}

Die Deutsche Gesellschaft für Allergologie und Klinische Immunologie e.V. (DGAKI), die Gesellschaft für Pädiatrische Allergologie und Umweltmedizin (GPA) und der Ärzteverband Deutscher Allergologen e.V. (AeDa) schreibt für 2015 zum ersten Mal den vom Arzneimittelhersteller ALKAbelló gestifteten Förderpreis Allergologie aus. Der Preis ist mit $30000 €$ dotiert und richtet sich an Studierende und Wissenschaftler aus dem deutschsprachigen Raum, die mit einer noch nicht abgeschlossenen Forschungsarbeit (Diplomarbeit, Dissertation, Habilitation oder andere Projekte) neue Ergebnisse erarbeiten, die die Versorgung allergiekranker Patienten deutlich verbessern. Wesentliches Kriterium bei der Vergabe des Preises ist daher auch die konsequente Orientierung am Patientennutzen. „Unser gemeinsames Ziel ist, die Versorgungssituation für Allergiker deutlich zu verbessern, daher wollen wir mit dem Preis wissenschaftliche und praxisorientierte Projekte auf dem Gebiet der Allergologie fördern“, so Dr. Kirsten Jung, Präsidentin des Ärzteverband Deutscher Allergologen. Bewerbungsschluss ist der 30. April 2015. Weitere Informationen hierzu finden Sie unter www.dgaki.de/wissenschaftspreise /weitere-wissenschaftspreise-andererinstitutionen.

Nach einer Mitteilung der DGAKI, Frankfurt a. $M$. 